03,09

\title{
Оптические переходы с остовных $d$-уровней арсенида галлия
}

\author{
(ㄱ Д.А. Перевощиков, В.В. Соболев \\ Удмуртский государственный университет, \\ Ижевск, Россия \\ E-mail: sobolev@uni.udm.ru
}

(Поступила в Редакцию 28 декабря 2016 г. В окончательной редакции 18 мая 2017 г.)

Усовершенствованным беспараметрическим методом объединенных диаграмм Арганда спектр диэлектрической проницаемости арсенида галлия в области $19-26 \mathrm{eV}$ разложен на 12 полос оптических переходов с определением их энергий максимумов и полуширин, а также сил осцилляторов. Значения сил осцилляторов полученных полос находятся в интервале от 0.0009 до 0.06 . В областях $19.2-21.3$ и $24-26 \mathrm{eV}$ спектр диэлектрической проницаемости был предварительно рассчитан на основе экспериментальных спектров отражения с использованием метода интегральных соотношений Крамерса-Кронига. Природа полученных полос переходов предложена по модели межзонных и экситонных переходов.

DOI: 10.21883/FTT.2018.03.45547.461

\section{1. Введение}

Соединения галлия группы $A^{3} B^{5}$ входят в число самых известных полупроводниковых материалов. Из-за широкого использования этой группы кристаллов в различных прикладных задачах (см., например, [1-3]) их электронной структуре посвящено много экспериментальных и теоретических работ [4-6]. Одним из самых „простых“ и распространенных методов экспериментального изучения электронной структуры неметаллов является измерение дисперсии оптических констант (отражения $R(E)$, поглощения $\alpha(E)$, диэлектрической проницаемости $\varepsilon(E)$ и т.д.). Данные таких измерений зачастую ограничены интервалом от края собственного поглощения до $\sim 7-10 \mathrm{eV}$, где спектры $R(E)$ и $\varepsilon(E)$ полупроводников наиболее интенсивны. Так как при возбуждении электронов светом интенсивность непрямых переходов на несколько порядков ниже интенсивности прямых, то такой метод позволяет зондировать зоны проводимости лишь при условии их параллельности валентным зонам в широкой области зоны Бриллюэна (ЗБ). Учитывая, что верхние валентные зоны (ВВ3) полупроводников имеют довольно сложную структуру [4,5], из чего условие параллельности валентных зон и зон проводимости в широкой области ЗБ выполняется крайне радко количество структур на зависимостях оптических функций до 7-10 eV не превышает шести-восьми [5-7].

Альтернативными методами экспериментального изучения расположения и особенностей энергетических уровней твердых тел являются спектроскопия характеристических потерь энергии электронов $[8,9]$ и использование лазеров со сверхкороткими импульсами [10]. В первом случае поток быстрых электронов при взаимодействии с исследуемым образцом может частично передавать свой волновой вектор электрону из валентной зоны, что приводит к интенсивным непрямым переходам. Вследствие этого появляется возможность исследовать структуру зон проводимости в более широкой области значений волнового вектора $\mathbf{k}$, чем при „простых“ оптических измерениях с чисто прямыми переходами. Во втором случае на основе генерации гармоник высокого порядка в поле сверхкоротких лазерных импульсов среднего инфракрасного диапазона предложен метод определения дисперсии нижней зоны проводимости (НЗП) твердых тел на примере $\mathrm{ZnSe}$. Существенным недостатком обоих этих методик является большая дороговизна используемых экспериментальных установок, что резко ограничивает их применение.

В силу этого интересным вариантом является исследование оптических свойств твердых тел в области переходов с остовных уровней. Дело в том, что остовные уровни полупроводников значительно у́же валентных зон (ширина полос менее $0.1 \mathrm{eV}$ ) и формируются за счет электронов лишь одного компонента соединения с одним типом симметрии, что дает большие преимущества. Во-первых, особенности получаемых оптических спектров зависят лишь от структуры зон проводимости и их симметрии. Так, например, по оптическим переходам из остовных $d$-зон можно судить о распределении $p$-состояний в зоне проводимости. Во-вторых, сильная локализация остовных уровней позволяет предположить существование сильных многочастичных эффектов, подобно сильноионным кристаллам с узкими ВВ3 [11]. В полупроводниках область переходов с остовных уровней обычно расположена при $E>12-15 \mathrm{eV}$. Поэтому для проведения экспериментальных измерений с хорошей точностью необходимо использовать синхротронные источники излучения. В данной области энергий точность таких измерений составляет $\sim 0.1-0.01 \mathrm{eV}$. Полученные экспериментальные результаты для полупроводников [12-19] показывают, что, вследствие перечисленных выше особенностей, спектры оптических функций в области переходов с остовных уровней содержат до восьми-девяти максимумов и ступенек. 
Первые существенные результаты по переходам с остовных $d$-уровней галлия для $\mathrm{GaAs}$ и $\mathrm{GaP}$ получены еще в 1963 г. [7]. Так как в качестве источника света использовалась аргоновая лампа, то для обоих кристаллов при энергиях от 17 до $26 \mathrm{eV}$ получена лишь одиночная широкая полоса, качественно объясняемая переходами из остовных $d$-уровней катионов. В течение последующих 50 лет в данной области энергий для всех четыpex соединений галлия группы $A^{3} B^{5}$ при помощи синхротронных источников излучения получены спектры поглощения [12], отражения [13,14], диэлектрической проницаемости [15-17], содержащие от двух до четырех максимумов и еще три-четыре ступеньки. В основном формирование этих структур качественно объяснялось спин-орбитальным расщеплением остовных состояний и экситонными переходами. При этом наиболее обширные и детальные экспериментальные результаты получены для GaAs [7,12-16].

Теоретически наиболее детально изучены особенности лишь трех-четырех ВB3 и НЗП, а также оптические переходы в области до $\sim 10 \mathrm{eV}$ [5,6,20-25]. Попытка теоретического описания оптических свойств кристаллов группы $A^{3} B^{5}$ при $E>17 \mathrm{eV}$ в одноэлектронном приближении представлена только для $\mathrm{GaAs}$ и $\mathrm{GaP}$ в одной работе [26]. Положение $d$-уровней было скорректировано по экспериментальным фотоэмиссионным данным. Полученные таким образом первые максимумы переходов из $d$-зон оказались смещенными на $\sim 1 \mathrm{eV}$ в область бо́льших энергий по сравнению с экспериментальными данными.

Цель настоящей работы состоит в моделировании полного набора оптических переходов кристалла GaAs в области 19-26 eV и определении их фундаментальных параметров: энергии максимумов и сил осцилляторов, а также в теоретическом анализе полученных данных на основе дополнительных теоретических расчетов межзонных переходов и учета возможного вклада метастабильных экситонов.

\section{2. Теория и метод расчета}

В стандартном способе определения набора оптических функций, в том числе, действительной $\varepsilon_{1}(E)$ и мнимой $\varepsilon_{2}(E)$ частей диэлектрической функции $\varepsilon(E)$ на основе экспериментальной зависимости $R(E)$ используют компьютерные пакеты программ с численной реализацией соотношений Крамерса-Кронига и аналитических формул связи между оптическими функциями. Данные методики нами подробно описаны [5,27] и обсуждены [11,28-30].

Разложение спектров $\varepsilon_{2}(E)$ на элементарные компоненты и определение энергий их максимумов $E_{j}$ и полуширин $H_{j}$ было выполнено в рамках усовершенствованного беспараметрического метода объединенных диаграмм Арганда (ОДА). Беспараметрический метод ОДА основан на предположении, что элементарная компонента оптического перехода имеет форму симметричного лоренцевского осциллятора [27,29-31]

$$
\begin{gathered}
\varepsilon_{1, j}(E)-1=\frac{\varepsilon_{2 \max , j} H_{j} E_{j}\left(E_{j}^{2}-E^{2}\right)}{\left(E_{j}^{2}-E^{2}\right)^{2}+H_{j}^{2} E^{2}}, \\
\varepsilon_{2, j}(E)=\frac{\varepsilon_{2 \max , j} H_{j}^{2} E_{j} E}{\left(E_{j}^{2}-E^{2}\right)^{2}+H_{j}^{2} E^{2}},
\end{gathered}
$$

где $\varepsilon_{2}$ max, ${ }$ - максимальное значение полосы $\varepsilon_{2, j}(E)$. Отличительная особенность такой изолированной полосы состоит в том, что ее диаграмма Арганда (зависимость $\left.\varepsilon_{2, j}=F\left(\varepsilon_{1, j}\right)\right)$ представляет собой идеальную окружность. Фундаментальная значимость этой окружности состоит в том, что ее параметры (диаметр, координаты ее центра) однозначно определяют параметры функций $\varepsilon_{1, j}$ и $\varepsilon_{2, j}: E_{j}, H_{j}$ и $\varepsilon_{2 \text { max,j. }}$. ОДА суммирует вклады множества окружностей отдельных полос, при этом вклад наиболее интенсивных проявляется структурно заметно лучше. Достраивая проявляющиеся неполные окружности на кривой ОДА до полных окружностей, можно, в итоге, получить все неизвестные параметры в уравнениях (1) и (2) для каждой выделенной полосы. Это позволяет шаг за шагом разложить весь спектр диэлектрической функции на отдельные компоненты без использования подгоночных параметров. Однако у этого метода есть весомый недостаток: хвосты лоренцевского распределения имеют физически необоснованную протяженность в широких областях энергии. Это противоречит теоретически ожидаемому формированию полосы в весьма ограниченной области энергии. Несмотря на слабость, эти хвосты могут приводить к сильному занижению интенсивности слабых полос переходов, вплоть до невозможности их выделения. Естественно было усовершенствовать беспараметрический метод ОДА заменой слабых боковых хвостов функций $\varepsilon_{2, j}(E)$ касательными к контуру полос на уровне $\varepsilon_{2 \max , j} / 4$, что эквивалентно замене начальной формы кривой лоренциана на гауссиан. Расчет соответственно изменяющейся функции $\varepsilon_{1, j}(E)$ проводился по соотношениям Крамерса-Кронига.

Зная контур элементарной полосы функции $\varepsilon_{2}(E)$, можно определить количество участвующих в переходах электронов в элементарной ячейке при формировании данной полосы (силу осциллятора):

$$
f_{j}=A \frac{\Omega}{z} \int_{a_{j}}^{b_{j}} E \varepsilon_{2, j}(E) d E,
$$

где $A=0.462 \mathrm{eV}^{-2} \cdot \mathrm{nm}^{-3}-$ константа, $\Omega-$ объем элементарной ячейки (в $\left.\mathrm{nm}^{3}\right), z=4-$ число функциональных единиц в элементарной ячейке, $a_{j}$ и $b_{j}-$ начало и конец элементарной полосы (в eV).

Для многих кристаллов известны методы воспроизведения интегрального спектра набором ряда полос с использованием 15-30 подгоночных параметров [20,31]. 
Существенное отличие примененной нами методики от известных многопараметрических моделей состоит в полном отсутствии подгоночных параметров.

Так как в нашей модели не заложена природа выделяемой полосы, то она может быть обусловлена межзонными переходами, экситонными переходами или их комбинацией в случае малой энергии связи экситонов относительно полуширины полосы. Для определения природы возникновения полученных полос мы будем опираться на модель межзонных переходов в рамках первопринципных методов расчета зон [32] и возможных метастабильных экситонов [4,5].

Расчеты зон и межзонных переходов проводились нами на основе теории функционала электронной плотности с использованием обменно-корреляционного потенциала GGA-PBESol [33], реализованной в пакете программ exciting [34]. Методы на основе GGAпотенциалов при больших достоинствах имеют три недостатка: 1) сильное занижение значения запрещенной зоны $[5,21,22,35,36], 2)$ некорректное описание оптических переходов в верхние зоны проводимости $[7,8], 3)$ завышение положений остовных уровней $[4,35,38-40]$. Для решения первой проблемы уже стало нормой простое смещение зон проводимости на постоянное значение энергии $\delta_{\text {gap }}$ до согласования теоретической и экспериментальной значений запрещенной зоны. Обычно данная поправка определяется по значению запрещенной зоны в точке $\Gamma\left(\delta_{\text {gap }}=\delta_{\text {gap }}(\Gamma)\right)$, но в случае кристаллов $A^{3} B^{5}$ дополнительно известны энергии запрещенной зоны в точках $L$ и $X$ [41]. Поэтому для более корректного определения погрешности в расчетах положения зон проводимости величину $\delta_{\text {gap }}$ будем определять по формуле

$$
\delta_{\text {gap }}=\left(\delta_{\text {gap }}(\Gamma)+\delta_{\text {gap }}(L)+\delta_{\text {gap }}(X)\right) / 3 \text {. }
$$

Для устранения второго недостатка наши расчеты ограничим рассмотрением переходов с $d$-уровней в три НЗП. Для существенного уменьшения третьей проблемы, как и в работе [26], введем поправку $\delta_{d}$ для интересующих нас остовных $3 d$-состояний $\mathrm{Ga}$. В результате общая поправка для межзонных переходов с участием остовных $d$-зон $\delta_{\text {gap }+d}$ будет представлять сумму $\delta_{\text {gap }}$ и $\delta_{d}$

$$
\delta_{\text {gap }+d}=\delta_{\text {gap }}+\delta_{d}
$$

В расчетах зон с учетом многочастичных методов в приближении GW [4] или более сложных обменнокорреляционных частей в самосогласованном уравнении состояния [36,39] плоские остовные уровни также в итоге лишь смещаются в область меньших значений энергии на постоянную величину, без заметного влияния на их дисперсию. Для межзонных переходов будем использовать следующие обозначения: переходы из $d^{5 / 2}$-состояний в зону проводимости $C_{j}$ обозначим $d(5) \rightarrow C_{j} \quad(j=1,2,3)$. Аналогично в случае с $d^{3 / 2}$ : $d(3) \rightarrow C_{j}$.
На основе результатов расчета зон можно также провести анализ возможных экситонных переходов, которые сопровождают межзонные переходы при энергии $E_{\mathrm{ex}}=\left(E_{C}-E_{V}\right)-E_{\text {bind }}$, где $E_{\mathrm{ex}}-$ энергия экситонного перехода, $E_{V}$ и $E_{C}$ - энергия валентной зоны и зоны проводимости, $E_{\text {bind }}-$ энергия связи электроннодырочной пары. Поскольку $E_{\text {bind }}$ определяется с большой погрешностью, то при обозначении энергии экситонного перехода будем ссылаться на энергию соответствующего межзонного перехода $E_{C V}=E_{C}-E_{V}+\delta_{\text {gap }+d}$ (в формуле мы уже учли необходимое смещение остовных зон и зон проводимости на $\delta_{d}$ и $\delta_{\text {gap }}$ соответственно). Сами экситонные переходы будем обозначать следующим образом: если переходы, например, происходят в точке $X$ и связаны с $d^{5 / 2}$-состояниями и зоной проводимости $C_{j}$, то обозначим их $X(5, j)$. Аналогично в случае с $d^{3 / 2}$. Для метастабильных экситонов, если они связаны с максимумом (ступенькой) в межзонных переходах $d(5) \rightarrow C_{j}$, обозначение примет вид $M E$ (peak (shoulder), $\left.d(5), C_{j}\right)$, или более кратко $M E(\mathrm{p}(\mathrm{sh}), 5, j)$. Аналогично в случае с $d(3) \rightarrow C_{j}$.

\section{3. Результаты расчетов}

На рис. 1 для GaAs приведены наши данные по расчетам зон вдоль некоторых направлений в ЗБ: остовные $d$-уровни $\left(\mathrm{Ga} 3 d^{5 / 2}\right.$ и $\left.\mathrm{Ga} 3 d^{3 / 2}\right)$ и три НЗП $\left(C_{1}\right.$, $C_{2}$ и $C_{3}$, причем $\left.E\left(C_{j+1}\right) \geq E\left(C_{j}\right)\right)$, за начало отсчета энергии принято положение максимума ВВ3 в центре ЗБ. Наши данные по величине спин-орбитального расщепления $d$-зон $\delta_{\mathrm{dsc}}=0.46 \mathrm{eV}$ хорошо согласуются с ранее рассчитанными в $[26,35,36]$ значениями, равными $\sim 0.4-0.46 \mathrm{eV}$, и со значениями, полученными экспериментально по данным фотоэмиссионных спектров $(0.4-0.453 \mathrm{eV}[42,43])$. Для сравнения наших результатов по зонной структуре с данными последних лет,

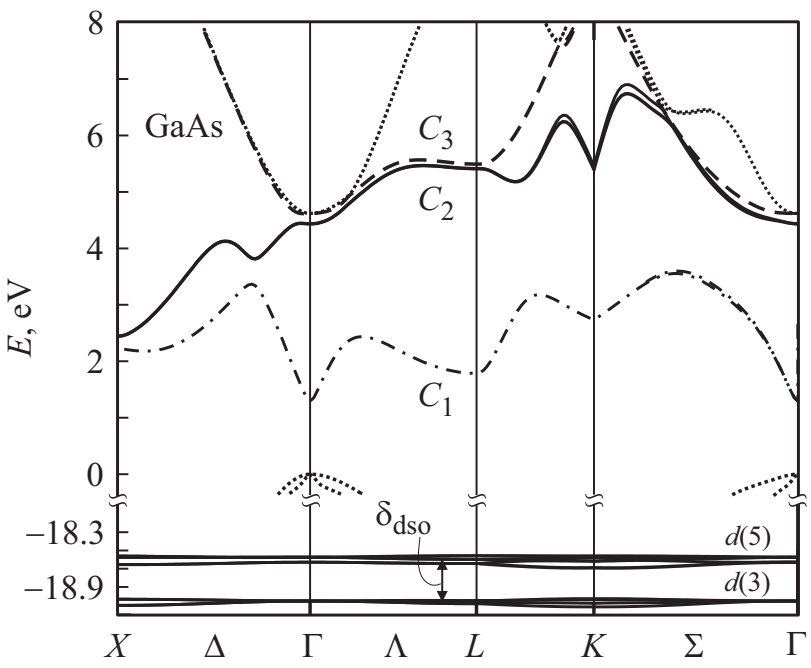

Рис. 1. Три нижние зоны проводимости $C_{1}, C_{2}, C_{3}$ и остовные $d$-зоны арсенида галлия $d(5)$ и $d(3)$. 
полученными первопринципными методами, зоны проводимости были смещены в область бо́льших энергий по формуле (4). Значение поправки $\delta_{\text {gap }}=0.97 \mathrm{eV}$. В результате, расхождения по энергии наших зон проводимости и данных работ $[21,22,36]$ не превышают $0.25 \mathrm{eV}$.

На основе рассчитанных нами зон были найдены парциальные вклады в $\varepsilon_{2}(E)$ переходов $d(5) \rightarrow C_{j}$ и $d(3) \rightarrow C_{j}(j=1,2,3)$ в три НЗП (рис. 2). Для определения параметра $\delta_{d}$ в формуле (5) использовались экспериментальные фотоэмиссионные данные положения остовных $d$-зон относительно максимума ВВ3 в точке $\Gamma$ из работы [43]. Значение поправки $\delta_{\mathrm{gap}+d}=4.87 \mathrm{eV}$. Расхождение положений рассчитанных нами максимумов межзонных переходов с данными работы [26] составило $\sim 0.1 \mathrm{eV}$.

Для рассматриваемой области энергий переходов известны экспериментальные спектры: поглощения поликристаллических пленок от 15 до $40 \mathrm{eV}$ при $300 \mathrm{~K}$ [12], отражения монокристаллов от 19.2 до $26 \mathrm{eV}$ при $300 \mathrm{~K}$ [13] и до $21.3 \mathrm{eV}$ при $14 \mathrm{~K}$ [14], $\varepsilon_{1}$ и $\varepsilon_{2}$ монокристаллов (эллипсометрические данные) от 16 до $26 \mathrm{eV}$ при $100 \mathrm{~K}$ [15] и от 19 до $24 \mathrm{eV}$ при $90 \mathrm{~K}$ [16]. Расчет фазы по данным работ $[13,14]$ осуществлялся методом Крамерса-Кронига (при $E<19.2 \mathrm{eV}$ использовался спектр $R(E)$ из работы [7]). Так как данные по спектрам отражения в $[13,14]$ приведены в относительных единицах, то для их нормировки мы использовали значения спектров отражения, полученные по известным спектрам диэлектрических проницаемостей из работ $[15,16]$. После этого по формулам связи были определены функции $\varepsilon_{1}(E), \varepsilon_{2}(E)$. При $E>26 \mathrm{eV}$ экспериментальные спектры поглощения в [12] не содержат ярко выраженных структур, поэтому рассмотрение ограничивалось интервалом энергии $19.2-26 \mathrm{eV}$. На рис. 2 приведены итоговые спектры действительной и мнимой частей диэлектрической проницаемости GaAs. От 19.2 до $21.2 \mathrm{eV}$ данные получены по спектру отражения из работы [14], от 21.2 до $24 \mathrm{eV}$ - по прямым эллипсометрическим измерениям [16], от 24 до $26 \mathrm{eV}-$ по спектру отражения [13]. Функция $\varepsilon_{2}(E)$ содержит три максимума (№ $4^{*}, 6^{*}, 9^{*}$ на рис. 2) и семь ступенек (№ $1^{*}-3^{*}, 5^{*}, 7^{*}, 8^{*}, 10^{*}$ на рис. 2). После разложения получено двенадцать полос оптических переходов (сплошные кривые на рис. 2). В таблице приведены их энергии максимумов и полуширины, силы осцилляторов, вычисленные по формуле (3), а также предполагаемые типы переходов, приводящих к их возникновению. В качестве погрешностей энергий максимумов $\Delta E_{\text {err }}$ можно принять заявленные в работах $[13,14,16]$ разрешающие способности экспериментальных установок в соответствующей области энергий, а именно от 19.2 до $21.2 \mathrm{eV}$ $\Delta E_{\mathrm{err}} \approx 0.01 \mathrm{eV}$, от 21.2 до $24 \mathrm{eV} \Delta E_{\text {err }} \approx 0.06 \mathrm{eV}$, при $E>24 \mathrm{eV} \Delta E_{\mathrm{err}} \approx 0.01 \mathrm{eV}$. Полученные значения сил осцилляторов находятся в интервале от 0.0009 до 0.06 . В $[13,14,16]$ нет сведений о погрешностях данных по интенсивности, поэтому для оценки погрешности значений $f_{j}$ обратимся к экспериментальным данным по спектрам поглощения поликристаллических пленок арсенида

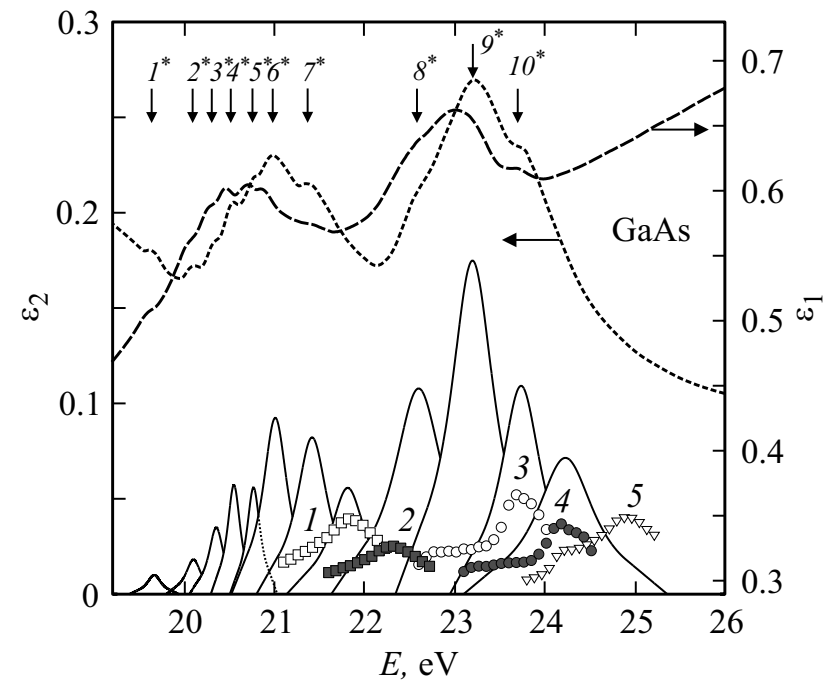

Рис. 2. Интегральные спектры $\varepsilon_{1}(E)$ и $\varepsilon_{2}(E) \mathrm{GaAs}$, энергии максимумов (№ $4^{*}, 6^{*}, 9^{*}$ ) и ступенек (№ $1^{*}-3^{*}, 5^{*}, 7^{*}$, $\left.8^{*}, 10^{*}\right) \varepsilon_{2}(E)$, полосы разложений $\varepsilon_{2}(E)$ (сплошные кривые), кривые парциальных вкладов межзонных переходов с $d$-состояний галлия в три НЗП: $d(5) \rightarrow C_{1}(1), d(3) \rightarrow C_{1}(2)$, $d(5) \rightarrow C_{2}(3), d(3) \rightarrow C_{2}(4), d(5) \rightarrow C_{3}(5)$.

галлия для данной области энергий [12]. Заявленная в данной работе погрешность определения интенсивности $\Delta I_{\text {err }}$ составляет $\sim 20 \%$. В $[13,14,16]$ измерения проводились на монокристаллах, поэтому $\Delta I_{\text {err }}$ должно быть заметно ниже. После выделения всех полос переходов среднее значение остатка составило $\sim 10 \%$ от интенсивности полос. Таким образом, можем ориентировочно принять, что погрешность значений сил осцилляторов равна $\Delta I_{\text {err }} \approx 20-25 \%$.

Принято считать, что в полупроводниках $A^{3} B^{5}$ НЗП сформирована $s$-состояниями. В теоретической работе [44] уточнено, что это верно лишь в центре ЗБ. Это предполагает, что любые оптические переходы, в том числе экситонные, из $d$-зон в НЗП в точке $\Gamma$ запрещены в дипольном приближении [5,27] и не должны проявляться на фоне существующих разрешенных переходов. В работе [24] при более детальном рассмотрении доказано, что в НЗП в точке $Г$ небольшой вклад дают $p$-состояния. Основываясь на этом, мы предполагаем, что при формировании полос № 2 и 4 заметный вклад могут давать также экситонные переходы $\Gamma(5,1)$ и $\Gamma(3,1)$ соответственно.

Полуширины выделенных полос переходов, начиная с шестой, становятся заметно больше, чем у предыдущих. Это, видимо, связано с влиянием сплошного спектра межзонных переходов из $d$-зон. Максимумы межзонных переходов $d(5) \rightarrow C_{1} \quad$ и $d(3) \rightarrow C_{1}$ возникают вследствие существования максимума в парциальном спектре $p$-состояний в зоне проводимости при $E=3.2 \mathrm{eV}$ (здесь и далее энергии максимумов и ступенек плотностей состояний приведены с учетом поправки на $\left.\delta_{\text {gap }}\right)$. Аналогично ступеньке и максимумам в переходах $d(5) \rightarrow C_{2}$ и 
Основные параметры разложения $\varepsilon_{2}(E)$ GaAs: полуширина $H_{j}$, сила осциллятора $f_{j}$, энергия максимума $E_{j}$, а также энергии $E_{C V}$ максимумов и ступенек (приведены в скобках) некоторых теоретических межзонных и возможных экситонных переходов. (Звездочкой обозначены энергии $E_{C V}$, полученные не по расчетам зон, а по экспериментальным данным о ширине запрещенной зоны [44] и положении $d$-зон [43])

\begin{tabular}{|c|c|c|c|c|c|c|c|c|c|}
\hline$j$ & $\begin{array}{l}H_{j} \\
\mathrm{eV}\end{array}$ & $f_{j} \cdot 10^{-2}$ & $\begin{array}{l}E_{j} \\
\mathrm{eV}\end{array}$ & $\begin{array}{c}\text { Энергия } E_{C V}, \mathrm{eV}, \\
\text { тип перехода }\end{array}$ & $j$ & $\begin{array}{l}H_{j} \\
\mathrm{eV}\end{array}$ & $f_{j} \cdot 10^{-2}$ & $\begin{array}{l}E_{j}, \\
\mathrm{eV}\end{array}$ & $\begin{array}{c}\text { Энергия } E_{C V}, \mathrm{eV}, \\
\text { тип перехода }\end{array}$ \\
\hline 1 & 0.19 & 0.09 & 19.66 & - & \multirow[t]{3}{*}{9} & \multirow[t]{3}{*}{0.67} & \multirow[t]{3}{*}{4.1} & \multirow[t]{3}{*}{22.60} & \multirow{3}{*}{$\begin{array}{c}(23.1), M E(\text { sh, 5, 2) } \\
22.3, d(3) \rightarrow C_{1}\end{array}$} \\
\hline 2 & 0.21 & 0.19 & 20.10 & $20.12^{*}, \Gamma(5,1)$ & & & & & \\
\hline 3 & 0.21 & 0.36 & 20.35 & $20.42^{*}, L(5,1)$ & & & & & \\
\hline 4 & 0.18 & 0.52 & 20.55 & $\begin{array}{l}20.57^{*}, \Gamma(3,1) \\
20.58^{*}, X(5,1)\end{array}$ & \multirow[t]{2}{*}{10} & \multirow[t]{2}{*}{0.59} & \multirow[t]{2}{*}{6.0} & \multirow[t]{2}{*}{23.20} & \multirow{2}{*}{$\begin{array}{c}23.7, M E(\mathrm{p}, 5,2) \\
(23.5), M E(\mathrm{sh}, 3,2) \\
(23.1), d(5) \rightarrow C_{2}\end{array}$} \\
\hline 5 & 0.18 & 0.53 & 20.77 & $20.87^{*}, L(3,1)$ & & & & & \\
\hline 6 & 0.34 & 1.6 & 21.01 & $\begin{array}{c}21.03^{*}, X(3,1) \\
21.05, X(5,2)\end{array}$ & \multirow[t]{2}{*}{11} & \multirow[t]{2}{*}{0.55} & \multirow[t]{2}{*}{3.5} & \multirow[t]{2}{*}{23.74} & \multirow{2}{*}{$\begin{array}{l}24.2, M E(\mathrm{p}, 3,2) \\
23.7, d(5) \rightarrow C_{2} \\
(23.5), d(3) \rightarrow C_{2}\end{array}$} \\
\hline 7 & 0.43 & 1.9 & 21.42 & $21.50, X(3,2)$ & & & & & \\
\hline & & & & $21.8, M E(\mathrm{p}, 5,1)$ & \multirow[t]{2}{*}{12} & \multirow[t]{2}{*}{0.78} & \multirow[t]{2}{*}{3.4} & \multirow[t]{2}{*}{24.2} & \multirow{2}{*}{$\begin{array}{c}24.2, d(3) \rightarrow C_{2} \\
(24.2), d(5) \rightarrow C_{3}\end{array}$} \\
\hline 8 & 0.47 & 1.4 & 21.8 & $\begin{array}{l}22.3, M E(\mathrm{p}, 3,1) \\
21.8, d(5) \rightarrow C_{1}\end{array}$ & & & & & \\
\hline
\end{tabular}

$d(3) \rightarrow C_{2}$ соответствуют ступенька и максимум при 4.5 и $5.1 \mathrm{eV}$ в спектре парциальной плотности р-состояний в зоне проводимости. Самая интенсивная выделенная полоса № 10 соответствует лишь широкой ступеньке в межзонных переходах $d(5) \rightarrow C_{2}$, а максимум переходов $d(5) \rightarrow C_{2}$ смещен в область бо́льших энергий на $\sim 0.5 \mathrm{eV}$ (рис. 2). Это не может быть причиной погрешности расчетов, поскольку, как было отмечено выше, отличие наших результатов от данных других теоретических работ составляет $\sim 0.1-0.25 \mathrm{eV}$. Поэтому можно предположить, что формирование данной полосы происходит в основном за счет экситонных переходов из $d$-зон $\left(E_{\text {bind }} \approx 0.3-0.5 \mathrm{eV}\right)$, связанных с максимумом в межзонных переходах $d(5) \rightarrow C_{2}(M E(\mathrm{p}, 5,2))$. Обобщением этого предположения является то, что экситонные переходы могут соответствовать всем максимумам и ступенькам в межзонных переходах из $d$-зон в две НЗП, что отражено в таблице.

\section{4. Выводы}

В настоящей работе на основе известных экспериментальных спектров отражения моделировалась диэлектрическая функция кристалла GaAs в интервале энергий от 19 до $26 \mathrm{eV}$, а спектр $\varepsilon_{2}(E)$ разложен на двенадцать компонент, определены энергии их максимумов и полуширины, а также силы осцилляторов. Значения сил осцилляторов находятся в интервале $(0.09-6) \cdot 10^{-2}$. Теоретически рассчитаны остовные $d$-зоны и межзонные переходы из них в три нижние зоны проводимости с учетом поправки на экспериментальные значения ширин запрещенных зон и положений $d$-уровней. Предположе- но, что выделенные двенадцать компонент спектра $\varepsilon_{2}(E)$ $\mathrm{GaAs}$ в основном обусловлены межзонными переходами в две нижние зоны проводимости, а также экситонными переходами.

\section{Список литературы}

[1] C. Hilsum. Semicond. Sci. Technol. 28, 015028 (2013).

[2] В.И. Стафеев. ФТП 44, 577 (2010).

[3] И. Акасаки. УФН 186, 504 (2016).

[4] F. Bechstedt. Many-body approach to electronic excitations. Concepts and applications. Springer-Verlag, Berlin (2015). $584 \mathrm{p}$.

[5] В.В. Соболев. Оптические свойства и электронная структура неметаллов. Том 1. Введение в теорию. Изд-во Института компьютерных исследований, М.-Ижевск (2012). $584 \mathrm{c}$.

[6] П. Ю, М. Кардона. Основы физики полупроводников. Физматлит, М. (2002). 560 с.

[7] H.R. Philipp, H. Ehrenreich. Phys. Rev. 129, 1550 (1963).

[8] U. Buchner. Phys. Status Solidi B 81, 227 (1977).

[9] В.В. Соболев, Е.А. Антонов, В.Вал. Соболев. ФТП 44, 1206 (2010).

[10] А.А. Ланин, А.М. Желтиков. Письма в ЖЭТФ 104, 475 (2016).

[11] В.В. Соболев, Д.А. Мерзляков, В.Вал. Соболев. ЖПС 83, 552 (2016).

[12] W. Gudat, E.E. Koch, P.Y. Yu, M. Cardona, C.M. Penchina. Phys. Status Solidi B 52, 505 (1972).

[13] D.E. Aspnes, M. Cardona, V. Saile, M. Skibowski, G. Sprussel. Solid State Commun. 31, 99 (1979).

[14] M. Skibowski, G. Sprussel, V. Saile. Appl. Opt. 19, 3978 (1980). 
[15] J. Barth, R.L. Johnson, M. Cardona, D. Fuchs, A.M. Bradshaw. In: Proc. 19th Int. Conf. Phys. Semicond. Polish Academy of Science, Warsaw (1988). V. 2. P. 885.

[16] O. Gunther, C. Janowitz, G. Jungk, B. Jenichen, R. Hey, L. Daweritz, K. Ploog. Phys. Rev. B 52, 2599 (1995).

[17] M. Rakel, C. Cobet, N. Esser, F. Fuchs, F. Bechstedt, R. Goldhahn, W.G. Schmidt, W. Schaff. Phys. Rev. B 77, 115120 (2008).

[18] P. Gori, M. Rakel, C. Cobet, W. Richter, N. Esser, A. Hoffmann, R.D. Sole, A. Cricenti, O. Pulci. Phys. Rev. B 81125207 (2010).

[19] K. Dorywalski, B. Andriyevsky, M. Piasecki, N. Lemee, A. Patryn, C. Cobet, N. Esser. J. Appl. Phys. 114, 043513 (2013).

[20] S. Adachi. Phys. Rev. B 35, 7454 (1987).

[21] А.В. Бакулин, С.Е. Кулькова. Изв. вузов. Физика 57, 7, 122 (2014).

[22] B.D. Malone, M.L. Cohen. J. Phys.: Condens. Matter, 25, 105503 (2013).

[23] M. Grüning, D. Sangalli, C. Attaccalite. Phys. Rev. B 94, 035149 (2016).

[24] В.Д. Дымников. ФТТ 43, 1957 (2001).

[25] A. Said, M. Debbichi, M. Said. Optik 127, 9212 (2016).

[26] P. Thiry, Y. Petroff, R. Pinchaux, J.R. Chelikowsky, M.L. Cohen. Solid State Commun. 20, 1107 (1976).

[27] В.В. Соболев, В.В. Немошкаленко. Методы вычислительной физики в теории твердого тела. Электронная структура полупроводников. Наукова думка, Киев (1988). 422 с.

[28] В.В. Соболев, В.Вал. Соболев, Д.В. Анисимов. ФТТ 57, 2415 (2015).

[29] В.В. Соболев. Оптические свойства и электронная структура моноуглеродных сред. Т. 1. Изд-во УдГУ, Ижевск (2016). $516 \mathrm{c.}$

[30] В.В. Соболев. Оптические свойства и электронная структура моноуглеродных сред. Т. 2. Изд-во УдГУ, Ижевск (2016). $724 \mathrm{c.}$

[31] В.В. Соболев. Оптические свойства и электронная структура неметаллов. Т. 2. Изд-во Института компьютерных исследований, М.-Ижевск (2012). 416 с.

[32] R.O. Jones. Rev. Mod. Phys. 87, 897 (2015).

[33] J.P. Perdew, A. Ruzsinszky, G.I. Csonka, O.A. Vydrov, G.E. Scuseria, L.A. Constantin, X. Zhou, K. Burke. Phys. Rev. Lett. 100, 136406 (2008).

[34] A. Gulans, S. Kontur, Ch. Meisenbichler, D. Nabok, P. Pavone, S. Rigamonti, S. Sagmeister, U. Werner, C. Draxl. J. Phys.: Condens. Matter 26, 363202 (2014).

[35] J. Wang, Y. Zhang, L.-W. Wang. Phys. Rev. B 92, 045211 (2015).

[36] A. Cakan, C. Sevik, C. Bulutay. J. Phys. D: Appl. Phys. 49, 085104 (2016).

[37] C.S. Wang, B.M. Klein. Phys. Rev. B 24, 3417 (1981).

[38] N.N. Anua, R. Ahmed, A. Shaari, M.A. Saeed, B.U. Haq, S. Goumri-Said. Semicond. Sci. Technol. 28, 105015 (2013).

[39] S. Sharma, J.K. Dewhurst, C. Ambrosch-Draxl. Phys. Rev. Lett. 95, 136402 (2005).

[40] В.В. Соболев, Д.А. Перевощиков. ФТП 49, 584 (2015).

[41] I. Vurgaftman, J.R. Meyer, L.R. Ram-Mohan. J. Appl. Phys. 89, 5815 (2001).

[42] N.J. Shevchik, J. Tejeda, M. Cardona. Phys. Rev. B 9, 2627 (1974).

[43] T. Miller, T.-C. Chiang. Phys. Rev. B 29, 7034 (1984).

[44] S.L. Richardson, M.L. Cohen, S.G. Louie, J.R. Chelikowsky. Phys. Rev. B 33, 1177 (1986). 